\title{
Editorial
}

\section{Propagation Phenomena and Transitions in Complex Systems 2013}

\author{
Ezzat G. Bakhoum, ${ }^{1}$ Cristian Toma, ${ }^{2}$ Carlo Cattani, ${ }^{3}$ and Ming $\mathrm{Li}^{4}$ \\ ${ }^{1}$ University of West Florida, Pensacola, FL 32514, USA \\ ${ }^{2}$ Faculty of Applied Sciences, University Politehnica of Bucharest, 70709 Bucharest, Romania \\ ${ }^{3}$ University of Salerno, 84084 Fisciano, Italy \\ ${ }^{4}$ School of Information Science and Technology, East China Normal University, No. 500 Dong-Chuan Road, \\ Shanghai 2002411, China
}

Correspondence should be addressed to Ezzat G. Bakhoum; ebakhoum@uwf.edu

Received 23 February 2014; Accepted 23 February 2014; Published 20 March 2014

Copyright (C) 2014 Ezzat G. Bakhoum et al. This is an open access article distributed under the Creative Commons Attribution License, which permits unrestricted use, distribution, and reproduction in any medium, provided the original work is properly cited.

The challenge in advanced engineering applications based on efficient mathematical models for propagation and transition phenomena can be noticed nowadays in many research fields. Fractal theory and special mathematical functions are used not only for the design of nanostructures but also for studying propagation in complex artificial networks. Differential geometry is adapted for solving nonlinear partial differential equations with very great number of variables for modelling propagation and transitions for different type of electromagnetic, acoustic, and optic waves. Commutative and/or additive consequences of quantum physics are used extensively in the design of long range transmission systems. Advanced mathematical tools connected to wavelets are recommended for biological phenomena. All these advanced engineering subjects require efficient mathematical models adapted for nonlinear propagation phenomena and for complex systems, when specific limitations are involved (very long distance propagation, fractal aspects and transitions in nanostructures, complex systems with great number of variables, and infinite spatiotemporal extension of material media). Using advanced mathematical tools for modeling propagation and transition phenomena, this special issue presents high qualitative and innovative developments for efficient mathematical approaches of Propagation Phenomena and Transitions in Complex Systems. Significant results were obtained for propagation of waves in advanced materials, dynamics of complex systems, efficient signal and image analysis based on fundamental mathematical and physical laws, and transitions in complex networks.

This special issue involves 13 original papers selected by the editors so as to present the most significant results in the previously mentioned topics. These papers are organised as follows.

(a) Five papers are on advanced mathematical approach for propagation and transmission in complex artificial networks: "Delay bound: fractal traffic passes through network servers" by M. Li et al., "Smoothing the sample autocorrelation of long-range-dependent traffic" by M. Li and W. Zhao, "An efficient patch dissemination strategy for mobile networks" by D. Zhao et al., "Attractor transformation by impulsive control in Boolean control network" by B. Gao et al., and "Identifying vulnerable nodes of complex networks in cascading failures induced by node-based attacks" by S. Li et al.

(b) Three papers are on specific methods for the analysis of propagation phenomena in physics: "Nonholonomic geometry of viscoanelastic media and experimental confirmation" by A. Ciancio and C. Cattani, "Acoustic response of a sinusoidally perturbed hard-walled duct" by S. D. Giudice and G. Bernasconi, and "Transient aspects of wave propagation connected with spatial coherence" by E. G. Bakhoum and C. Toma.

(c) Two papers are on accurate and efficient mathematical models for transition in biological systems: "Legendre wavelets method for solving fractional population growth 
model in a closed system" by M. H. Heydari et al. and "A class of solutions for the hybrid kinetic model in the tumor-immune system competition" by C. Cattani and A. Ciancio.

(d) Three papers are on mathematical tools for analyzing mathematical complexity: "Golden ratio phenomenon of random data obeying von Karman spectrum" by M. Li and W. Zhao, "Content-based image retrial based on Hadoop" by D. Yin and D. Liu, and "Essay on fractional Riemann-Liouville integral operator versus Mikusinskis” by M. Li and W. Zhao.

Ezzat G. Bakhoum Cristian Toma

Carlo Cattani Ming Li 


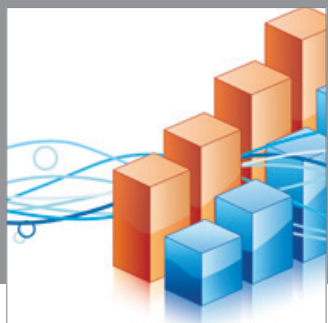

Advances in

Operations Research

mansans

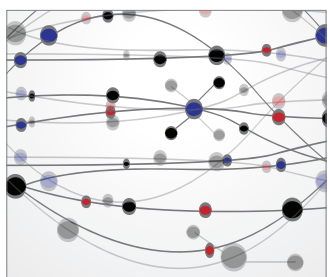

The Scientific World Journal
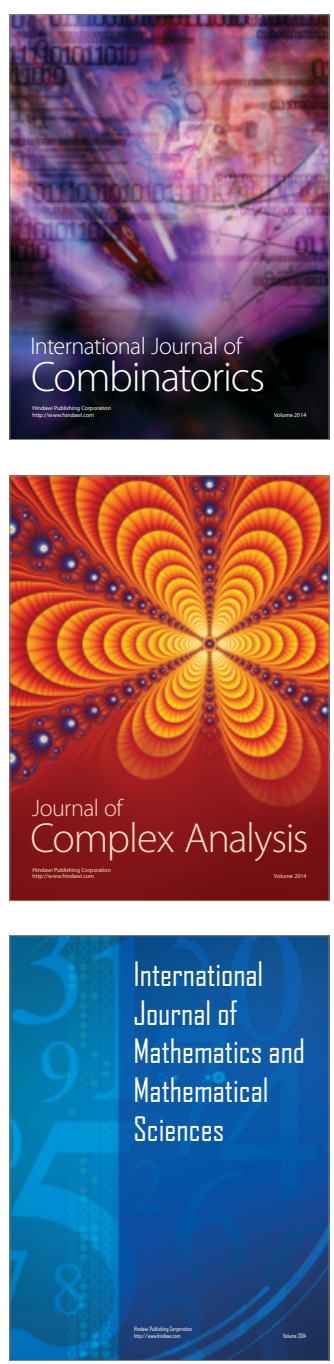
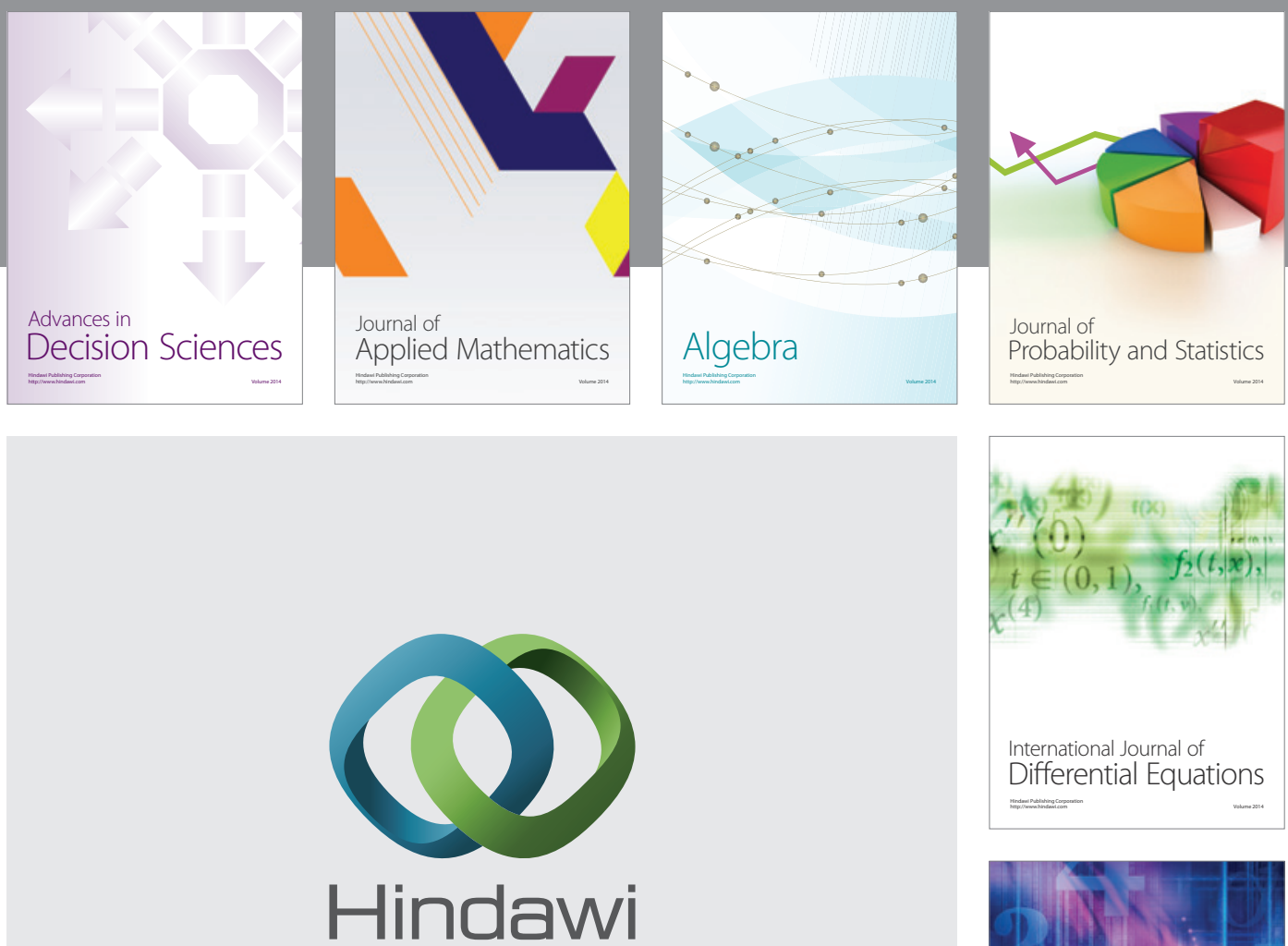

Submit your manuscripts at http://www.hindawi.com
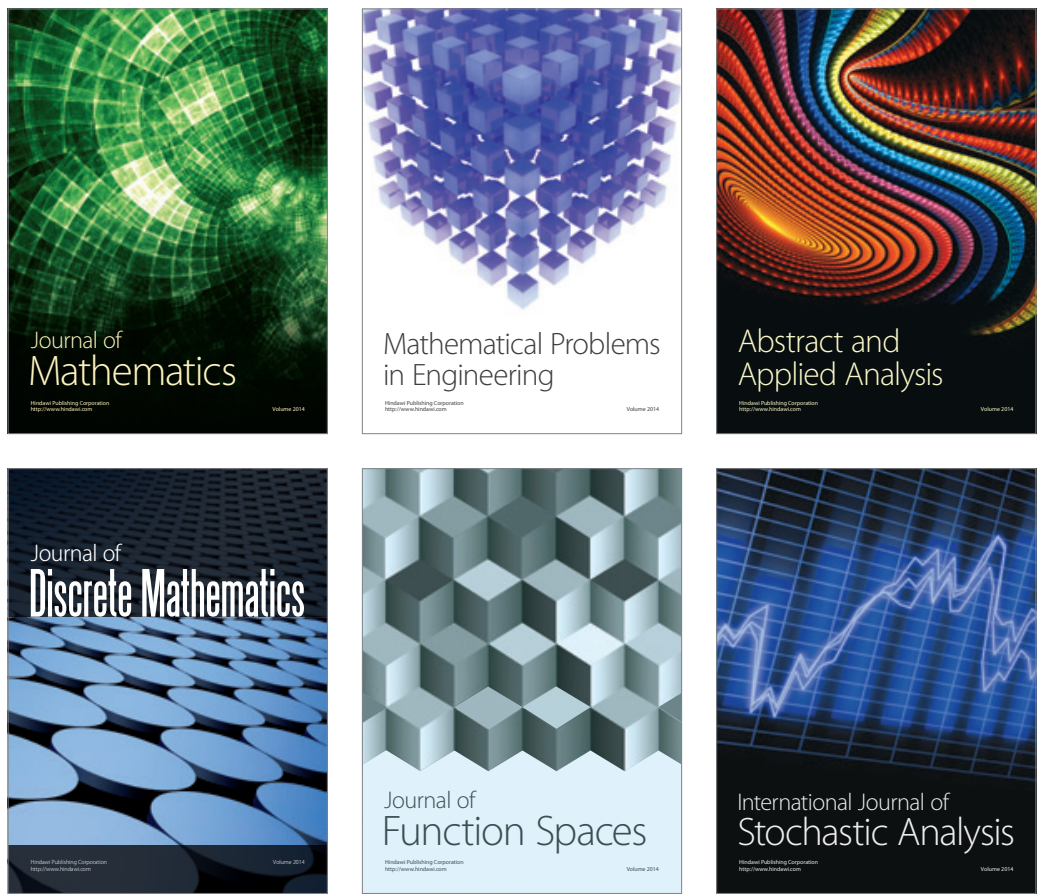

Journal of

Function Spaces

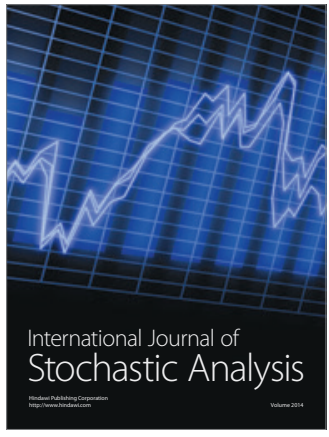

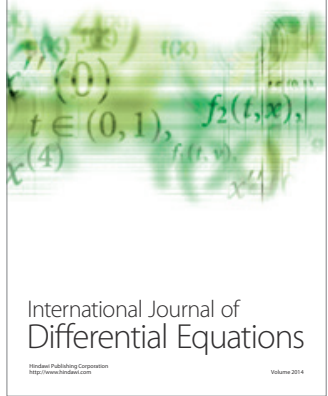
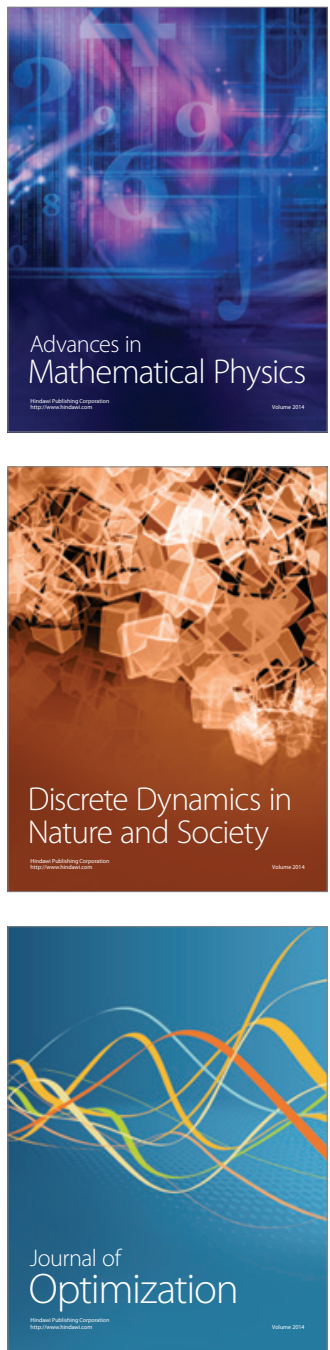